\title{
Estructura factorial y propiedades psicométricas de la Time Management Behavior Scale (TMBS) en población universitaria colombiana*
}

\section{Factorial Structure and Psychometric Properties of the Time Management Behavior Scale (TMBS) in Colombian College Students}

Recepción: 03 Marzo 2013 | Aprobación: 09 Diciembre 2016

\author{
Angélica Garzón Umerenkova ${ }^{\mathrm{a}}$ \\ Universidad El Bosque, Colombia \\ Rafael García Ros \\ Universidad de Valencia, España \\ Francisco Pérez-GonzÁlez \\ Universidad de Valencia, España
}

a Autora de correspondencia. Correo electrónico:
agarzonu@gmail.com

Para citar este artículo: Garzón Umerenkova, A., García Ros, R., \& Pérez-González, F. (2017). Estructura factorial y propiedades psicométricas de la Time Management Behavior Scale (TMBS) en población universitaria colombiana. Universitas Psychologica, 16(1), 1-10. http://dx.doi.org/10.11144/Javeriana.ups y16-1.efpp

\section{RESUMEN}

Se investigó la estructura factorial de la Time Management Behavior Scale (TMBS) en universitarios colombianos. La gestión del tiempo es un importante predictor del logro estudiantil y clave para la disminución de la deserción universitaria. Participaron 494 estudiantes de primer año de nueve programas en dos universidades de la ciudad de Bogotá. Se comprueba una estructura multidimensional de cuatro subescalas que explicaron el $42.68 \%$ de la varianza total. Se obtuvieron índices de consistencia interna por encima de 0.7 en tres factores y una fiabilidad global de 0.84. Coincidiendo con investigaciones previas, frente a los hombres, las mujeres obtienen puntuaciones superiores en dos de las subescalas, y los estudiantes de las carreras de educación presentan mejores puntuaciones en Percepción del Control del Tiempo que los estudiantes de ingeniería. Se comparan los resultados en Colombia con la prueba norteamericana y la española.

Palabras clave

estudiantes universitarios; gestión del tiempo; psicometría; validación.

\section{ABSTRACT}

The factorial structure of the Time Management Behavior Scale (TMBS) was investigated in Colombian college students. Time management is an important predictor of the academic achievements and is key in reducing college desertion. 494 first year students from nine different programs and two universities in Bogotá city participated. A multidimensional structure of four sub scales that explain $42.68 \%$ of the variance was verified. Indexes of internal consistency above 0.7 in three factors and a global reliability of 0.84 were obtained. In accordance with previous research, women obtain higher scores against men in two of the obtained sub scales, and the students of Education programs score better in "Perception of time control" than engineering students. The results obtained in Colombia are compared with the North American and Spanish test.

Keywords

college students; time management; psychometrics; validation. 
De acuerdo a los datos de la Organización para la Cooperación y el Desarrollo Económicos (OCDE), en promedio, un $30 \%$ de los universitarios abandona sus estudios. Sin embargo, hay diferencias importantes entre países; mientras que en Dinamarca o Japón las tasas de deserción son de menos del $25 \%$, en Hungría o Suecia son del 40 \% y son aún más elevadas en Latinoamérica, donde la deserción se sitúa en torno al $57 \%$ de los estudiantes (OCDE, 2013).

De este modo, aunque entre el 2000 y el 2010, Latinoamérica tuvo un aumento de la cobertura en las matrículas universitarias cercano al $40 \%$, se debe avanzar también en la disminución de las tasas de deserción en la región (UNESCO, 2013). Como en el resto de Latinoamérica, en Colombia se ha producido en los últimos años un considerable incremento del número de universitarios, pasando de un $27 \%$ en el 2004 al $42 \%$ en el 2013 (Velásquez, 2013). Sin embargo, la deserción acumulada para el nivel de estudios universitario es del $46.6 \%$ en el quinto año de carrera, y el $18.5 \%$ del total de los abandonos se produce en el primer semestre (Ministerio de Educación Nacional, 2016). Si se incluyeran los datos de la formación técnica profesional y tecnológica, estas cifras aumentarían.

Por otra parte, en los últimos años las condiciones personales de aprendizaje relacionadas con la autorregulación académica han ganado terreno sobre variables sociodemográficas tradicionales en el estudio de la predicción del éxito estudiantil y en la prevención de un potencial fracaso (Robbins, Oh, Le, \& Button, 2009; Rosário et al., 2014). Entre los factores individuales que influyen en la deserción académica universitaria, la capacidad de gestionar adecuadamente el tiempo es uno de los predictores más importantes del logro estudiantil (García-Ros \& Pérez-González, 2011; George, Dixon, Stansal, Lund, \& Pheri, 2008; Krumrei, Elizabeth, Newton, Kim, \& Wilcox, 2013; Pehlivan, 2013; Xu, 2010). Al parecer, durante el primer año universitario, tanto la gestión del tiempo como la autoeficacia son buenos predictores del rendimiento de los estudiantes al final del curso, pero solo la gestión del tiempo continúa siendo predictor del logro académico un año después (Kitsantas, Winsler, \& Huie, 2008).

La gestión del tiempo puede definirse como "conductas que buscan lograr un uso efectivo del tiempo, mientras se ejecutan ciertas actividades dirigidas a una meta" (Claessens, Van Eerde, Rutte, \& Roe, 2007, p. 262). Este constructo se considera un componente importante de la autorregulación académica por su relación con la planificación, el establecimiento de metas, el automonitoreo y otros factores metacognitivos y motivacionales (Gray, 2015; Zimmerman, 1996). Tiene una relación inversa con la procrastinación académica (Garzón \& Gil, 2017), que a su vez es considerada la "quintaesencia" del fallo autorregulatorio (Steel, 2007). Por lo tanto, el desarrollo de esta competencia se relaciona con el fomento de la autonomía y la capacidad de "aprender a aprender" de los estudiantes, aspectos determinantes en la vida universitaria.

Algunos autores han encontrado mejores habilidades de gestión del tiempo en estudiantes mujeres (Durán-Aponte \& Pujol, 2013; Kaya, Kaya, Palloş, \& Küçük, 2012; Pehlivan, 2013). Otros estudios señalan alguna ventaja en esta habilidad entre los estudiantes trabajadores de mayor edad (Forbus, Newbold, \& Mehta, 2010).

Desde una perspectiva práctica y preventiva, y aunque las causas del abandono de los estudios superiores son numerosas y complejas, la intervención temprana sobre algunas competencias con demostrada influencia sobre el logro académico (tales como el adecuado manejo del tiempo) pueden aumentar la probabilidad del éxito de los estudiantes durante su primer año de ingreso a la universidad.

Dada la importancia de este constructo, distintos autores han desarrollado cuestionarios específicos para medir gestión del tiempo, entre los que destacan el Time Structure Questionnaire (TSQ) (Bond \& Feather, 1988), el Time Management Questionnaire (TMQ) (Britton \& Tesser, 1991) y la Time Management Behavior Scale (TMBS) (Macan, Shahani, Dipboye, \& Phillips, 1990). La TMBS presenta algunas ventajas frente a otros cuestionarios; es 
la prueba más empleada en la investigación para medir de manera específica y estandarizada este constructo (Claessens et al., 2007), y ofrece una visión más concreta sobre lo que los estudiantes hacen para gestionar su tiempo.

Finalmente, se señala que en los últimos años hay un creciente reporte de estudios sobre la gestión del tiempo en entornos académicos fuera del ámbito anglosajón (p. ej., Azar \& Zafer, 2013; Dura\#n-Aponte \& Pujol, 2013; Liu, Rijmen, MacCann, \& Roberts, 2009; Nadinloyi, Hajloo, Garamaleki, \& Sadeghi, 2013; Olowookere, Alao, Odukoya, Adekeye, \& Agbude, 2015), destacando la progresiva necesidad de instrumentos de evaluación y de investigación al respecto.

En consecuencia, el propósito principal del presente estudio es analizar la dimensionalidad y las propiedades psicométricas de la Time Management Behavior Scale (Macan et al., 1990) en población universitaria colombiana de primer año. Diversos estudios han constatado la validez factorial de la TMBS que, en general, reproducen la estructura original con algunos cambios en la ubicación o reducción de los ítems; igualmente, se han obtenido con éxito versiones abreviadas o modificadas del cuestionario (Azar \& Zafer, 2013; Liu et al., 2009), fuera del contexto de habla hispana.

En coherencia con estos antecedentes, se espera que los resultados de la aplicación de la TMBS en Colombia mantengan el sentido de la prueba original, aunque con movimientos o/y reducción en algunos de los ítems, conservando adecuados indicadores validez y confiabilidad.

\section{Método}

\section{Participantes}

Se realizó un muestreo por conveniencia con la participación de dos instituciones universitarias de la ciudad de Bogotá (Colombia). La muestra estuvo constituida por 494 universitarios con edades comprendidas entre los 16 y 46 años; en total, fueron 127 hombres y 367 mujeres, 442 inscritos en I semestre y 49 en II semestre (primer año). De estos, 347 participantes pertenecían a la jornada diurna y 147 a la nocturna, en las carreras de Psicología (291 estudiantes), Educación Bilingüe (16), Pedagogía (31), Ingeniería de Sistemas (39), Ingeniería Electrónica (19), Administración de Empresas (21), Finanzas y Comercio Exterior (53), Negocios Internacionales (10) y Marketing (11).

\section{Instrumentos y variables}

Se elaboró un cuestionario ad hoc para el presente estudio con el objetivo de determinar las variables sociodemográficas de los estudiantes (edad, género, carrera, jornada diurna o nocturna, dedicación al estudio completa o parcial).

El TMBQ (Time Management Behavior Questionnaire) es una prueba constituida por 34 ítems que miden gestión del tiempo académico, utilizando una escala de respuesta tipo Likert con cinco anclajes, en la que 1 significa nunca y 5 siempre. La escala original aplicada en este trabajo fue desarrollada por Macan (1994), integrando cuatro subescalas o factores que dieron cuenta del $72 \%$ de su varianza: 1) Fijar metas y prioridades, 2) Herramientas para la gestión del tiempo, 3) Percepción del control del tiempo y 4) Preferencia por la desorganización.

\section{Procedimiento}

Se partió de la adaptación al castellano de la TMBS desarrollada por García-Ros y PérezGonzález (2012), sobre la que se realizó una revisión lingüística en busca de términos confusos, ambiguos o de poco uso en la cultura colombiana, por parte de un traductor profesional, tres psicólogos y cinco universitarios a través de entrevista semiestructurada. A partir de ello, se realizaron pequeñas modificaciones en algunas palabras y expresiones en 15 de los 34 ítems. Los ítems modificados y las instrucciones se presentan al final de este artículo en el Anexo A. (Error 1: La referencia: Anexo A está ligada a un elemento que ya no existe) 
La aplicación de las pruebas se efectuó de forma colectiva en aulas de informática de las universidades participantes, durante los meses de febrero y marzo del 2011, a través de una plataforma Web construida para el estudio; durante la aplicación el investigador siempre estuvo presente para resolver las dudas de los participantes. La participación en el estudio fue voluntaria y, con anterioridad a la aplicación, se obtuvo el consentimiento informado de los estudiantes o el asentimiento de sus padres o tutores, para el caso de los menores de edad. Los análisis se realizaron con el programa estadístico SPSS versión 21.

\section{Resultados}

\section{Descriptivos}

La Tabla 1 presenta los estadísticos descriptivos básicos de los ítems, destacando un comportamiento relativamente homogéneo en las puntuaciones medias de los ítems, situándose en niveles próximos a la media teórica (3). Por otro lado, también puede observarse que existe una tendencia a que las respuestas se encuentren ligeramente por encima de la media, con una asimetría con valores negativos.

\section{Análisis factorial}

Con el objetivo de investigar la estructura factorial subyacente a las respuestas ofrecidas por los sujetos, se efectuó un Análisis Factorial de Componentes Principales con rotación VARIMAX. Tras constatar que cuatro de los ítems no cumplían con los requisitos previamente establecidos (el ítem 27 presentó saturaciones superiores a 0.4 en dos factores, mientras que los ítems 4, 10 y 11 no presentaron saturación alguna superior a 0.4), se efectuó un segundo análisis factorial con los 30 ítems restantes. Los análisis iniciales del mismo constatan la adecuación de los datos para la aplicación de este tipo de análisis, dado que tanto la prueba de esfericidad de Bartlett (c2 (435) = 3793.16, p <
0.001) como la prueba de adecuación muestral de Kaiser-Meyer-Olkin (0.86) presentan valores adecuados.

Para la extracción del número de factores, se consideraron criterios teóricos y empíricos. Se tuvo en cuenta tanto la estructura factorial destacada por Macan et al. (1990) como la obtenida en España por García-Ros y PérezGonzález (2012), con cuatro factores en ambos casos. Además de este criterio teórico, la consideración conjunta de la prueba de Kaiser (valores propios por encima de 1), junto al scree test (gráfica de sedimentación) y la interpretabilidad de las estructuras resultantes (Gorsuch, 1983) generó una estructura de cuatro factores que explican el $42.86 \%$ de la varianza de los datos, mostrando una estrecha correspondencia con la estructura original y la obtenida en España.

\section{TABLA 1}

Descriptivos de los ítems del TMBS

\begin{tabular}{|c|c|c|c|c|}
\hline Item & Media & $\begin{array}{l}\text { Desviación } \\
\text { estándar }\end{array}$ & Asimetria & Curtosis \\
\hline tmbl & 3.86 & 0.99 & -0.628 & -0.077 \\
\hline $\mathrm{tmb2}$ & 3.45 & 1.089 & -0.32 & -0.551 \\
\hline $\mathrm{tmb} 3$ & 3.07 & 1.499 & -0.078 & -1.428 \\
\hline $\operatorname{tmb} 4$ & 2.82 & 1.04 & 0.145 & -0.363 \\
\hline tmb5 & 3.37 & 1.028 & -0.336 & -0.434 \\
\hline tmb6 & 2.94 & 1.1 & -0.008 & -0.716 \\
\hline tmb7 & 3.25 & 1.096 & -0.224 & -0.561 \\
\hline tmb8 & 3.9 & 1.177 & -0.902 & -0.141 \\
\hline $\operatorname{tmb} 9$ & 3.73 & 0.995 & -0.566 & -0.002 \\
\hline tmb10 & 3.39 & 1.063 & -0.235 & -0.558 \\
\hline tmbl1 & 3.13 & 1.299 & -0.186 & -1.048 \\
\hline tmb12 & 4.07 & 1.2 & -1.049 & -0.102 \\
\hline tmbl3 & 3.34 & 1.126 & -0.348 & -0.549 \\
\hline $\operatorname{tmb} 14$ & 3.49 & 1.313 & -0.495 & -0.881 \\
\hline tmb15 & 3.44 & 1.011 & -0.345 & -0.292 \\
\hline tmb16 & 4.07 & 1.079 & -0.967 & -0.008 \\
\hline tmb17 & 3.67 & 0.96 & -0.631 & 0.269 \\
\hline tmb18 & 2.85 & 1.421 & 0.104 & -1.31 \\
\hline tmb19 & 3.52 & 1.133 & -0.22 & -0.93 \\
\hline tmb20 & 3.66 & 1.169 & -0.445 & -0.78 \\
\hline $\mathrm{tmb21}$ & 3.71 & 1.069 & -0.605 & -0.16 \\
\hline $\operatorname{tmb} 22$ & 2.79 & 1.546 & 0.204 & -1.474 \\
\hline $\mathrm{tmb} 23$ & 3.54 & 1.207 & -0.313 & -0.954 \\
\hline tmb24 & 2.54 & 1.166 & 0.27 & -0.859 \\
\hline tmb25 & 2.21 & 1.313 & 0.707 & -0.79 \\
\hline $\operatorname{tmb} 26$ & 3.6 & 1.245 & -0.506 & -0.787 \\
\hline $\operatorname{tmb} 27$ & 2.92 & 1.157 & -0.11 & -0.86 \\
\hline tmb28 & 3.05 & 1.373 & -0.054 & -1.22 \\
\hline tmb29 & 3.06 & 1.144 & 0.024 & -0.654 \\
\hline tmb30 & 3.56 & 1.137 & -0.438 & -0.581 \\
\hline tmb31 & 3.6 & 1.154 & -0.558 & -0.416 \\
\hline $\mathrm{tmb} 32$ & 3.25 & 1.139 & -0.293 & -0.607 \\
\hline tmb33 & 3.1 & 1.214 & -0.202 & -0.885 \\
\hline tmb34 & 3.78 & 1.102 & -0.683 & -0.213 \\
\hline
\end{tabular}

Nota: Frente a cada ítem se indican los estadísticos descriptivos. Fuente: elaboración propia. 
La Tabla 2 presenta los resultados básicos del análisis factorial (saturación factorial y comunalidad de los ítems), destacando adicionalmente su convergencia con la estructura original y la obtenida con población española. El primer factor (valor propio de 4.2 y que explica el $14 \%$ de la varianza de los datos) integra trece ítems y se corresponde con la dimensión de Establecimiento de Objetivos y Prioridades. El segundo factor (valor propio de 3.74 y que explica el $12.45 \%$ de la varianza) integra siete ítems y corresponde a la escala original de Herramientas para la Gestión. El tercer factor (valor propio de 2.71 y que explica el $9.04 \%$ de la varianza de los datos) se asocia con la subescala original de Percepción del Control del Tiempo. Por último, el cuarto factor (valor propio de 2.13 y que explica el $7.18 \%$ de la varianza) está constituido por cuatro ítems y corresponde a la escala original Preferencias por la Desorganización. Por otro lado, las dos últimas columnas de la Tabla 2 permiten comparar la estructura obtenida en población colombiana con la destacada en población anglosajona (Macan, 1994) y en población española (GarcíaRos \& Pérez-González, 2012).

\section{TABLA 2}

Estructura factorial del TMBS con pesos factoriales tras rotación ortogonal (VARIMAX)

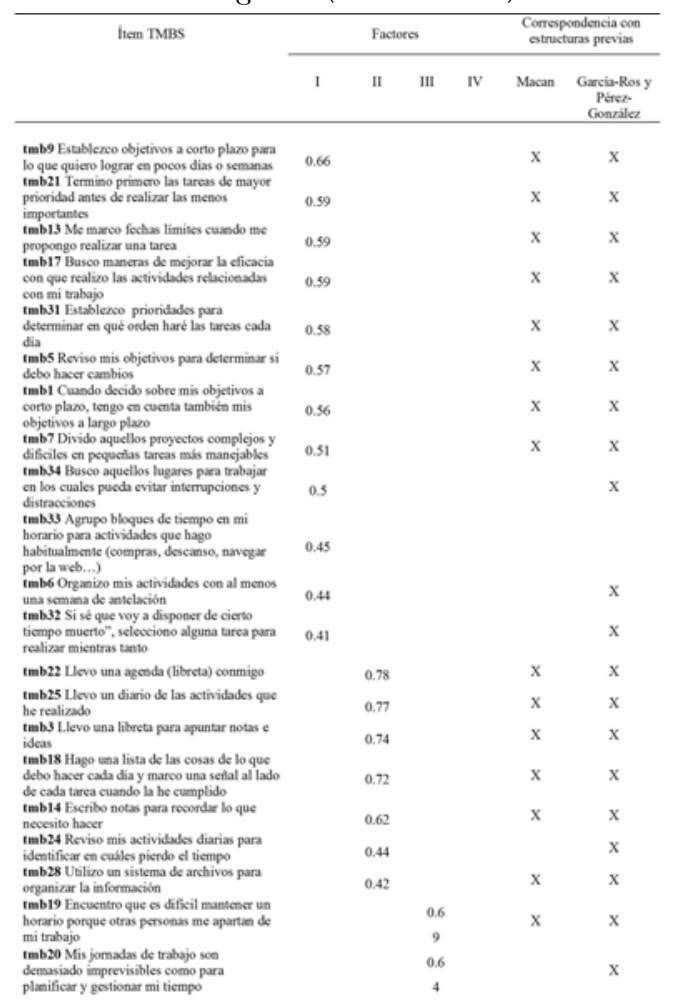

Nota: Factor 1: Establecimiento de metas y prioridades. Factor 2: Herramientas para la gestión del tiempo. Factor 3: Percepción del control del tiempo. Factor 4: Preferencias por la desorganización. Los ítems se presentan en orden de pertenencia a cada factor. Fuente: elaboración propia.

\section{Confiabilidad}

La fiabilidad de las subescalas se determinó a partir del alpha de Cronbach. Los valores obtenidos corresponden a 0.82 para Establecimiento de metas y prioridades, 0.82 para Herramientas para la gestión del tiempo, 0.71 para Percepción de control del tiempo y 0.67 para Preferencias por la desorganización. Finalmente, considerando los 30 ítems que componen la versión colombiana del TMBS (véase Anexo A), la fiabilidad de la prueba en su conjunto es de 0.84. (Error 2: La referencia: Anexo A está ligada a un elemento que ya no existe) 
Niveles de asociación entre subescalas

Se obtienen valores significativos medios entre el primer y segundo factor $\left(\mathrm{r}=0.68 \mathrm{p}<0.001^{* *}\right)$, así como entre el tercero y el cuarto $(r=0.45 \mathrm{p}<$ $0.001 * *)$, similares a los obtenidos con la versión original del instrumento y en población española. Establecimiento de metas y prioridades también presenta relaciones significativas, aunque de carácter muy reducido, con Preferencias por la desorganización $(\mathrm{r}=-0.16, \mathrm{p}<0.001 * * *)$ y con Percepción de control del tiempo $(\mathrm{r}=-0.11, \mathrm{p}<$ $0.05 *)$.

Análisis diferenciales en función de tipo de titulación y sexo

Para determinar los posibles efectos del sexo y del tipo de titulación sobre la gestión del tiempo, se efectuó un MANOVA factorial, donde las variables dependientes corresponden a las puntuaciones en las dimensiones del TMBS y las independientes al sexo y al tipo de titulación (Educación, Ingenierías y Sociales). Los resultados muestran la existencia de efectos significativos -aunque con un efecto reducidosobre la gestión del tiempo tanto de la variable sexo, $\mathrm{L}=0.97, \mathrm{~F}(4,446.000)=3.57, \mathrm{p}<$ 0.001, $\eta^{2}=0.03$, como de tipo de titulación, $\mathrm{L}$ $=0.95, \mathrm{~F}(8,892.000)=3.67, \mathrm{p}<0.001, \eta^{2}=$ 0.05, aunque no así de la interacción sexo*tipo titulación, $\mathrm{L}=0.99, \mathrm{~F}(8,892.000)=0.59, \mathrm{p}$ $<0.79, \eta^{2}=0.01$. Con el objetivo de clarificar los resultados del MANOVA se efectuaron en primer lugar los correspondientes ANOVA para cada dimensión de gestión del tiempo, cuyos resultados se presentan en la Tabla 3. Así, se constata la significancia de los efectos de la variable sexo en Herramientas para la gestión $\mathrm{F}(1,449)=5.4, \mathrm{p}<0.05^{*}, \eta^{2}=0.02$, así y en Preferencias por la desorganización $-\mathrm{F}$ (1, $449)=9.14, \mathrm{p}<0.01 *, \eta^{2}=0.03-$ Y de forma más específica, los análisis de contraste o comparación de medias por sexo y programa académico a posteriori, indicaron que las mujeres presentan valores significativamente superiores respecto de los varones en Herramientas para la gestión (diferencia medias $=2.35, \mathrm{p}<0.01)$ y significativamente inferiores en Preferencias por la desorganización (diferencia medias $=-1.47$, $\mathrm{p}$ $<0.01)$.

Por otro lado, el tipo de titulación muestra efectos significativos en Percepción de control del tiempo $-\mathrm{F}(2,449)=4.35, \mathrm{p}<0.05^{*}, \eta^{2}=$ $0.02-$. En este caso, los estudiantes de Educación manifiestan valores significativamente mejores en este factor respecto a los estudiantes de Ingeniería (diferencia medias $=-2.35, \mathrm{p}<0.01$ ).

\section{TABLA 3}

ANOVA sobre las dimensiones de gestión del tiempo: efectos principales e interacción entre sexo y tipo de titulación

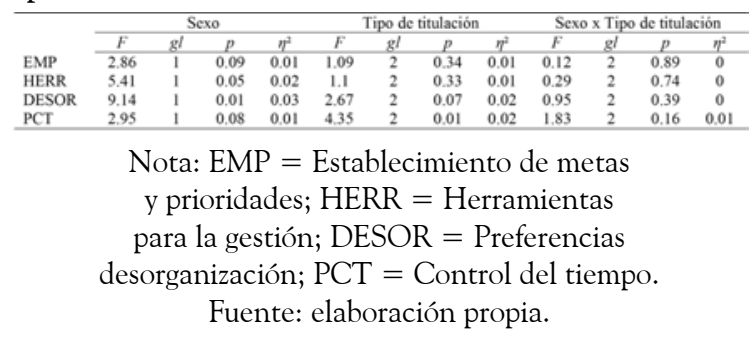

\section{Discusión}

Atendiendo al principal objetivo de este estudio, caracterizar la estructura factorial y las propiedades psicométricas de la TMBS (Macan et al., 1990), para su potencial utilización con estudiantes universitarios de primer año en Colombia, los resultados indican que la TMBS está conformada por cuatro subescalas relativamente independientes que explican el $42.68 \%$ de la varianza de los datos, presentando niveles de consistencia interna adecuados.

Los resultados preliminares de la TMBS en estudiantes universitarios colombianos, coincidiendo con los resultados de la investigación previa en contexto anglosajón y español, muestran que el significado de los factores que constituyen la gestión del tiempo son:

a) Establecimiento de metas y prioridades: evalúa la predisposición del estudiante para seleccionar y priorizar las tareas en función de la obtención de sus objetivos. 
b) Herramientas para la gestión del tiempo: mide el uso de técnicas/herramientas para la gestión eficaz del tiempo (p. ej., uso de agenda y listas de chequeo, entre otros).

c) Percepción de control del tiempo: valora el grado en el que el estudiante percibe que controla su tiempo de forma eficaz en la gestión de los eventos diarios. Puntuaciones superiores en este factor indican mayor percepción de falta de control.

d) Preferencias por la desorganización: estima la forma en que el estudiante organiza sus tareas académicas y mantiene un entorno de estudio estructurado. En este caso, puntuaciones elevadas en el factor indican preferencia por realización de actividades sin planificación ni organización previa.

En coherencia con la investigación previa (Durán-Aponte \& Pujol, 2013; Kaya et al., 2012; Pehlivan, 2013), los resultados en Colombia también señalan que las mujeres evidencian mejores puntuaciones que los varones en gestión del tiempo, específicamente en las dimensiones relativas a Herramientas y Percepción de control.

Los resultados de la presente investigación proporcionan apoyo empírico a la estructura factorial del cuestionario y, en último término, apoyo a la estructura multidimensional del constructo gestión del tiempo. Los resultados de la aplicación de la TMBS en Colombia, al igual que la versión norteamericana y española, parecen ajustarse a los cuatro factores anteriormente definidos. Estos resultados, en términos generales, comprueban la evidencia previa sobre las subescalas que componen la TMBS presentada por Macan et al. (1990), quienes originalmente definieron conceptual y empíricamente los elementos constitutivos de este instrumento.

Posteriores investigaciones tendrán que abordar el estudio psicométrico de los ítems ambiguos o poco discriminativos con respecto a las cuatro dimensiones de la Escala, de manera que sean reformulados o bien definitivamente eliminados.

Por último, cabe resaltar que las correlaciones entre las subescalas fueron moderadas o bajas, mostrando niveles de asociación similares a los destacados en la investigación previa. Lo que aporta evidencia adicional de que la gestión del tiempo es de naturaleza multifactorial e indica, a nivel práctico, la posible conveniencia de usar no solo la prueba en su totalidad, sino también sus dimensiones, tanto para la investigación como para la evaluación educativa de esta competencia.

Dadas las elevadas tasas de abandono en nuestro país en el primer semestre de ingreso a la universidad, la información aportada por la TMBS puede ser usada para direccionar los recursos humanos y técnicos hacia el apoyo a los estudiantes en la mejora de sus competencias de gestión del tiempo. Sin embargo, una de las limitaciones de la presente investigación es haber contado solo con estudiantes de primer y segundo semestres en la caracterización psicométrica de la prueba.

Próximos estudios deberán abordar el valor predictivo de los resultados de la TMBS sobre el rendimiento académico en aras de aportar evidencia de validez predictiva de la prueba, así como estudios que relacionen sus resultados con otras pruebas de gestión del tiempo, para aportar evidencia de su validez convergente.

Es recomendable continuar en un proceso de adaptación de pruebas de comprobada fiabilidad y validez a nuestro contexto latinoamericano, tanto para mejorar los procesos de evaluación asociados con la deserción académica universitaria como para fomentar la investigación transcultural, en esta área de estudio de la psicología educativa.

\section{Agradecimientos}

Se agradece al Dr. José Antonio Sánchez por el apoyo para la realizacion de este trabajo, a la Facultad de Psicología de la Universidad El Bosque por la financiación brindada y a la Fundación Universitaria 
Empresarial de la Cámara de Comercio de Bogotá (Uniempresarial) por su participación.

\section{Referencias}

Azar, S., \& Zafer, S. (2013). Confirmatory factor analysis of Time Management Behavior Scale: Evidence from Pakistan. Interdisciplinary Journal of Contemporary Research in Business, 4(12), 946-959. doi.org/10.2466/pr0.80.1.225-226

Bond, M. J., \& Feather, N. T. (1988). Some correlates of structure and purpose in the use of time. Journal of Personality and Social Psychology, 55(2), 321-329. doi:10.1037/0022-3514.55.2.321

Britton, B. K., \& Tesser, A. (1991). Effects of time-management practices on college grades. Journal of Educational Psychology, 83(3), 405-410. doi:10.1037/0022-0663.83.3.405

Claessens, B. J. C., van Eerde, W., Rutte, C. G., \& Roe, R. A. (2007). $A$ review of the time management literature. Personnel Review, 36(2), 255-276. doi:10.1108/00483480710726136

Durán-Aponte, E., \& Pujol, L. (2013). Manejo del tiempo académico en jóvenes que inician estudios en la Universidad Simón Bolívar. Revista Latinoamericana de Ciencias Sociales, Niñez y Juventud, 11 (1), 93-108.

Forbus, P., Newbold, J., \& Mehta, S. (2010). A study of non traditional and traditional students in terms of their time management behaviors, stress factors, and coping strategies. Proceedings of the Academy of Educational Leadership, 15(2), 67-71.

García-Ros, R., \& Pérez-González, F. (2011). Validez predictiva e incremental de las habilidades de autorregulación sobre el éxito académico en la universidad. Revista de Psicodidáctica, 16, 231-250.

García-Ros, R., \& Pérez-González, F. (2012). Spanish version of the Time Management Behavior Questionnaire for university students. The Spanish
Journal of Psychology, 15(03), 1485-1494. doi:10.5209/rev_sjop.2012.v15.n3.39432

Garzón, A., \& Gil, J. (2017). Propiedades psicométricas del Time Management Behavior Questionnaire (TMBS) en universitarios. Revista Electrónica de Investigación Educativa, 19(3). (En prensa).

George, D., Dixon, S., Stansal, E., Lund, S., \& Pheri, T. (2008). Time diary and questionnaire assessment of factors associated with academic and personal success among university undergraduates. Journal of American College Health, 56(6), 706-715.

Gorsuch, R. (1983). Factor analysis. Hillsdale, NJ: Erlbaum.

Gray, L. E. (2015). Exploring time management as a construct of self regulated learning in first-year college students taking online classes (Tesis doctoral inédita). Capella University, Minneapolis.

Kaya, H., Kaya, N., Palloş, A. Ö., \& Küçük, L. (2012). Assessing time-management skills in terms of age, gender, and anxiety levels: A study on nursing and midwifery students in Turkey. Nurse Education in Practice, 12(5), 284-288. doi:10.1016/ j.nepr.2012.06.002

Kitsantas, A., Winsler, A., \& Huie, F. (2008). Self-regulation and ability predictors of academic success during college: A predictive validity study. Journal of Advanced Academics, 20(1), 42-68.

Krumrei, M., Elizabeth, J., Newton, F., Kim, E., \& Wilcox, D. (2013). Psychosocial factors predicting first-year college student success. Journal of College Student Development, 54(3), 247-266. doi:10.1353/csd.2013.0034

Liu, O. L., Rijmen, F., MacCann, C., \& Roberts, R. (2009). The assessment of time management in middle-school students. Personality and Individual Differences, 47(3), 174-179. doi:10.1016/j.paid.2009.02.018

Macan, T. H. (1994). Time management: Test of a process model. Journal of Applied Psychology, 79(3), 381-391. doi: 10.1037/0021-9010.79.3.381 
Macan, T. M., Shahani, C., Dipboye, R., \& Phillips, A. (1990). College students' time management: Correlations with academic performance and stress. Journal of Educational Psychology, 82(4), 760-768.

Colombia, Ministerio de Educación Nacional. (2016). Sistema para la Prevención de la Deserción de la Educación Superior (SPADIES). Tasa de deserción por semestre. Recuperado de http://spadies.mineducacion.gov.co/spa dies/consultas_predefinidas.html?2

Nadinloyi, K. B., Hajloo, N., Garamaleki, N. S., \& Sadeghi, H. (2013). The study efficacy of time management training on increase academic time management of students. Procedia - Social and Behavioral Sciences, 84, 134-138. doi: 10.1016/j.sbspro.2013.06.523

Organización para la Cooperación y el Desarrollo Económicos. (2013). Education at a glance 2013. OECD indicators. OECD Publishing. doi: 10.1787/eag-2013-en

Olowookere, E., Alao, A., Odukoya, J., Adekeye, O., \& Ade' Agbude, G. (2015). Time management practices, character development and academic performance among university undergraduates. Creative Education, 6, 79-86. doi:10.4236/ ce.2015.61007

Pehlivan, A. (2013). The effect of the time management skills of students taking financial accounting course on their course grades and grade point averages. International Journal of Business and Social Science, 4(5), 196-199.

Robbins, S. B., Oh, I. -S., Le, H., \& Button, C. (2009). Intervention effects on college performance and retention as mediated by motivational, emotional, and social control factors: Integrated meta-analytic path analyses. Journal of Applied Psychology, 94(5), 1163-1184. doi:10.1037/a0015738

Rosário, P., Pereira, A. S., Högemann, J., Nunez, A. R., Figueiredo, M., Núñez, J. C., ... Gaeta, M. L. (2014). Autorregulación del aprendizaje: una revisión sistemática en revistas de la base Scielo. Universitas Psychologica, 13(2). doi:10.11144/javeriana.upsy13-2.aars

Steel, P. (2007). The nature of procrastination: A meta-analytic and theoretical review of quintessential self-regulatory failure. Psychological Bulletin, 133(1), 65-94.

Organización de las Naciones Unidas para la Educación, la Ciencia y la Cultura. (2013). Situación educativa de América Latina y el Caribe: hacia la educación de calidad para todos al 2015 (Informe). Santiago de Chile: OREALC/UNESCO.

Velásquez, O. (12 de octubre de 2013). Las 'descuadernadas' cifras de la educación superior. Dinero. Recuperado de http://www.dinero.com/opinion/column istas/articulo/las-descuadernadas-cifras-ed ucacion-superior/189362

$\mathrm{Xu}$, J. (2010). Predicting homework time management at the secondary school level: A multilevel analysis. Learning and Individual Differences, 20(1), 34-39. doi: 10.1016/j.lindif.2009.11.001

Zimmerman, B. (1996). Enhancing student academic and health functioning: a self regulatory perspective. School Psychology Quarterly, 11(1), 47-66.

\section{ANEXO A}

Se presentan las instrucciones e ítems definitivos para Colombia. La prueba presenta la numeración original de la prueba, pero se eliminaron cuatro ítems de la escala original (4, 10, 11 y 27). Para facilitar su eventual aplicación y calificación, frente a cada ítem se señala si es de calificación inversa (I) y a qué subescala pertenece; Fijar metas (FM), Herramientas para la gestión del tiempo $(\mathrm{H})$, Preferencias por la Desorganización (PD) y Percepción del control del tiempo (PC).

ESCALA DE COMPORTAMIENTOS DE GESTIÓN DEL TIEMPO (TMBS)

¿Hasta qué punto describe cada una de las afirmaciones en las siguientes páginas tus actividades y experiencias en relación con el 
estudio? Indica con qué exactitud cada frase te describe, eligiendo una de las alternativas de la siguiente escala. No hay respuestas correctas ni erróneas. Por favor responde a todas las preguntas.

(1) nunca / (2) pocas veces / (3) algunas veces / (4) habitualmente / (5) siempre

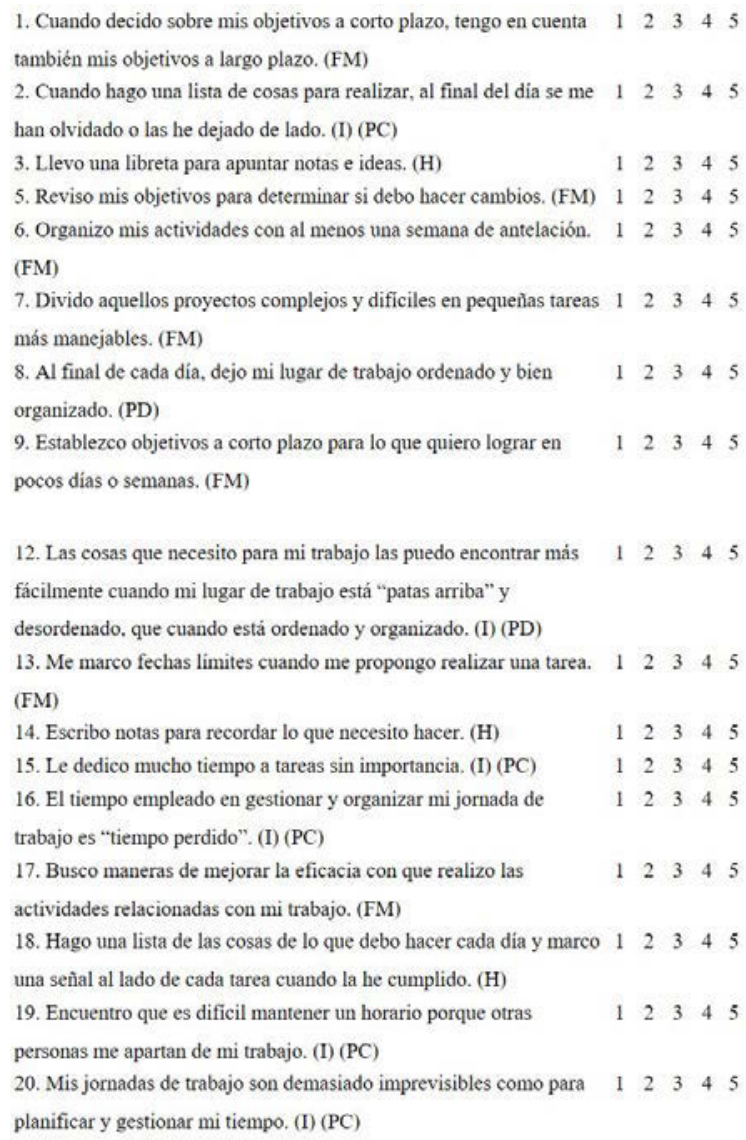

| Universitas Psychologica | V. i6 | No. i | Enero-Marzo | 2017 |

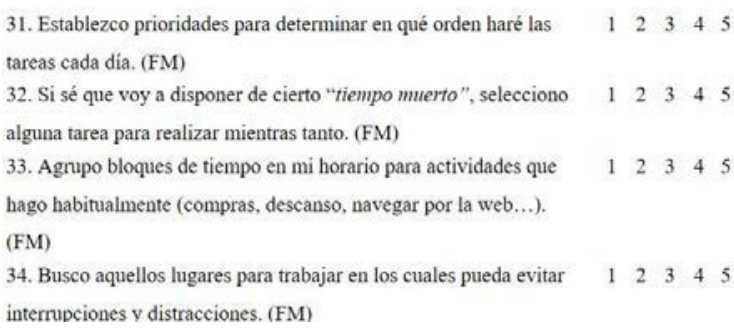

\section{Notas}

* Artículo de investigación. 